\title{
A NOTE ON $K$-COMMUTATIVITY OF MATRICES
}

\author{
EDMOND DALE DIXON AND NANCY WONG
}

\begin{abstract}
It is the purpose of this paper to find in terms of parameters the most generai matrix $X$ which is $K$-commutative with respect to a given matrix $A$. The proofs will yield a method of rational construction for such a matrix $X$.
\end{abstract}

Introduction. In $1936 \mathrm{~W}$. E. Roth [4] made an extensive investigation of $K$-commutativity of matrices. His theorems gave conditions for existence in terms of elementary divisors. It is the purpose of this paper to find in terms of parameters the most general matrix $X$ which is $K$ commutative with respect to a given matrix $A$. The proofs will yield a method of rational construction for such a matrix $X$.

Let $A$ and $X$ be $n \times n$ matrices with elements from a field $F$. We denote $A X-X A$ by $[A, X]$ or $[A, X]_{1}$. Then $[A, X]_{t}=\left[A,[A, X]_{t-1}\right]$. The matrix $X$ is said to be $K$-commutative with respect to $A$ if $[A, X]_{k}=0$. It is easily seen from the above equations that we may assume that $A$ has been reduced to rational canonical form. Furthermore, we may assume without loss of generality that the rational canonical form for $A$ is $\operatorname{diag}\{C(f), C(g)\}$. In our theorems we find the blocks of $X$ after it has been partitioned conformaliy with $A$. Thus we give conditions for the existence and give the construction of the most general matrix $X_{k}$ satisfying $C(f) X_{k}=X_{k} C(g)+$ $X_{k-1}, C(f) X_{k-1}=X_{k-1} C(g)+X_{k-2}, \cdots, C(f) X_{1}=X_{1} C(g)$ where $C(f)$ and $C(g)$ are companion matrices of the polynomials $f(x)$ and $g(x)$ and where $f(x)$ divides $g(x)$ or $g(x)$ divides $f(x)$.

The methods used were developed by W. V. Parker [1] in his investigation of the equation $A X=X B$.

Two-commutative case. We now study the equation

$$
C(f) Y=Y C(g)+X, \text { where } C(f) X=X C(g) \text {. }
$$

THEOREM 1. If $C(f)$ and $C(g)$ are companion matrices of the polynomiais $f(x)=x^{m}-\sum_{i=1}^{m} a_{i} x^{i-1}$ and $g(x)$ of degrees $m$ and $n$ respectively over the field $F$, then the $m \times n$ matrices $Y$ and $X$, over $F$, satisfy the equation

Received by the editors June 28, 1971.

AMS 1969 subject classifications. Primary 1263.

Key words and phrases. $K$-commutative matrices, canonical form, companion matrix, minimum polynomial.

c American Mathematical Society 1972 
$C(f) Y=Y C(g)+X$, where $C(f) X=X C(g)$, if and only if the row's of $X$ and $Y$ are $U, U C(g), \cdots, U C^{m-1}(g)$ and $V, V C(g)+U, \cdots, V C^{m-1}(g)+$ $(m-1) U C^{m-2}(g)$ respectively, where $U$ and $V$ are $1 \times n$ matrices satisfying the following conditions:

$$
V f[C(g)]+U f^{\prime}[C(g)]=0 \text { and } U f[C(g)]=0 .
$$

Proof. Parker [1] has shown that the rows of $X$ are $U, U C(g), \cdots$, $U C^{m-1}(g)$ where $U f[C(g)]=0$. If the rows of $Y$ are $V_{1}, V_{2}, \cdots, V_{m}$, then the rows of $C(f) Y$ are $V_{2}, V_{3}, \cdots, V_{m}$ and $\sum_{i=1}^{m} a_{i} V_{i}$. The rows of $Y C(g)+$ $X$ are $V_{1} C(g)+U, V_{2} C(g)+U C(g), \cdots, V_{m} C(g)+U C^{m-1}(g)$. Setting the corresponding rows of (1) equal gives

$$
\begin{aligned}
& V_{2}=V_{1} C(g)+U, \\
& V_{3}=V_{2} C(g)+U C(g)=V_{1} C^{2}(g)+2 U C(g),
\end{aligned}
$$

.

$$
\begin{gathered}
V_{m}=V_{m-1} C(g)+U C^{m-2}(g)=V_{1} C^{m-1}(g)+(m-1) U C^{m-2}(g), \\
\sum_{i=1}^{m} a_{i} V_{i}=V_{m} C(g)+U C^{m-1}(g)=V_{1} C^{m}(g)+m U C^{m-1}(g),
\end{gathered}
$$

then

$$
\begin{aligned}
a_{1} V_{1}+a_{2} V_{2}+\cdots+a_{m} V_{m}= & a_{1} V_{1}+a_{2}\left[V_{1} C(g)+U\right]+\cdots \\
& +a_{m}\left[V_{1} C^{m-1}(g)+(m-1) U C^{m-2}(g)\right] \\
= & V_{1} C(g)+m U C^{m-1}(g) .
\end{aligned}
$$

Transposing and collecting the coefficients of $V_{1}$ and the coefficients of $U$ gives

$$
\begin{aligned}
& V_{1}\left[C^{n}(g)-\left(a_{1} I+a_{2} C(g)+\cdots+a_{m} C^{m-1}(g)\right)\right] \\
& \quad+U\left[m C^{m-1}(g)-\left(a_{2} I+2 a_{3} C(g)+\cdots+(m-1) a_{m} C^{m-2}(g)\right)\right]=0 .
\end{aligned}
$$

That is $V_{1} f[C(g)]+U f^{\prime}[C(g)]=0$. Since all the steps of this proof are reversible, the converse holds.

It should be noted that if $g(x)$ divides $f(x)$, then $V_{1} f[C(g)]=0$ and $U f[C(g)]=0$ and $V_{1}$ is arbitrary. If $f(x)$ divides $g(x)$, a $1 \times n$ matrix different from zero exists satisfying (3).

Equations (2) are satisfied if and only if

and

$$
V[C(g)] f[C(g)]+U[C(g)] f^{\prime}[C(g)]=0
$$

$$
U[C(g)] f[C(g)]=0 .
$$


Thus $V$ and $U$ must be such that

(4) $V(x) f(x)+U(x) f^{\prime}(x)=g(x) q(x)$ and $U(x) f(x)=g(x) k(x)$

where $k(x)$ and $q(x)$ are arbitrary polynomials such that the degrees of $g(x) k(x)$ and $g(x) q(x)$ are not greater than $m+n-1$. Therefore the $1 \times n$ matrices $V$ and $U$ are obtained through their associated polynomials.

$K$-commutative case. We now study the system

$$
\begin{aligned}
& C(f) X_{k}=X_{k} C(g)+X_{k-1}, \\
& C(f) X_{k-1}=X_{k-1} C(g)+X_{k-2}, \\
& \dot{\cdot} \\
& \cdot \\
& C(f) X_{2}=X_{2} C(g)+X_{1},
\end{aligned}
$$

where $C(f) X_{1}=X_{1} C(g)$ and where $C(f)$ and $C(g)$ are companion matrices of the polynomials $f(x)=x^{m}-\sum_{i=1}^{m} a_{i} x^{i-1}$ and $g(x)$ of degrees $m$ and $n$ respectively.

THEOREM 2. Let $C(f)$ and $C(\dot{g})$ be as given in (5). Then the set of $m \times n$ motrices $\left\{X_{1}, X_{2}, \cdots, X_{k}\right\}$, over $F$, satisfy (5) if and only if the rows of $X_{1}, X_{2}, \cdots, X_{k}$ are

$$
\begin{aligned}
& \left(\begin{array}{l}
0 \\
0
\end{array}\right) U_{1},\left(\begin{array}{l}
1 \\
0
\end{array}\right) U_{1} C(g), \cdots,\left(\begin{array}{c}
m-1 \\
0
\end{array}\right) U_{1} C^{m-1}(g) \\
& \left(\begin{array}{l}
0 \\
0
\end{array}\right) U_{2},\left(\begin{array}{l}
1 \\
0
\end{array}\right) U_{2} C(g)+\left(\begin{array}{l}
1 \\
i
\end{array}\right) U_{1}, \cdots,\left(\begin{array}{c}
m-1 \\
0
\end{array}\right) U_{2} C^{m-1}(g) \\
& + \\
& \vdots \\
& \left(\begin{array}{l}
0 \\
0
\end{array}\right) U_{k},\left(\begin{array}{l}
1 \\
0
\end{array}\right) U_{k} C(g)+\left(\begin{array}{c}
1 \\
1
\end{array}\right) U_{k-1}, \cdots,\left(\begin{array}{c}
m-1 \\
0
\end{array}\right) U_{k} C^{m-2}(g) \\
& +\left(\begin{array}{c}
m-1 \\
1
\end{array}\right) U_{k-1} C^{m-2}(g)+\cdots+\left(\begin{array}{c}
m-1 \\
k-1
\end{array}\right) U_{1} C^{m-k}(g)
\end{aligned}
$$

successively, where $U_{1}, U_{2}, \cdots, U_{k-1}$, and $U_{k}$ are a set of $1 \times n$ matrices 
satisfying the following equations:

$$
\begin{aligned}
& U_{k} f[C(g)]+U_{k-1} f^{\prime}[C(g)]+\cdots+U_{1} \frac{f^{(k-1)}[C(g)]}{(k-1) !}=0, \\
& U_{k-1} f[C(g)]+U_{k-2} f^{\prime}[C(g)]+\cdots+U_{1} \frac{f^{(k-2)}[C(g)]}{(k-2) !}=0, \\
& \cdot \\
& \cdot \\
& \cdot \\
& U_{1} f[C(g)]=0 .
\end{aligned}
$$

Proof. We have shown that if $K=2$ then the rows of $X_{2}$ are $\left(\begin{array}{l}0 \\ 0\end{array}\right) U_{2}$, $\left(\begin{array}{l}1 \\ 0\end{array}\right) U_{2} C(g)+\left(\begin{array}{l}1 \\ 1\end{array}\right) U_{1}, \ldots,\left(\begin{array}{c}m-1 \\ 0\end{array}\right) U_{2} C^{m-1}(g)+\left(\begin{array}{c}m-1 \\ 1\end{array}\right) U_{1} C^{m-2}(g)$ where

$$
U_{2} f[C(g)]+U_{1} f^{\prime}[C(g)]=0 \text { and } U f[C(g)]=0 .
$$

We now proceed by induction to construct the rows of $X_{k}$ for arbitrary $K$.

Assume that if $K=p-1$ the rows of $X_{p-1}$ are

$$
\begin{array}{r}
\left(\begin{array}{l}
0 \\
0
\end{array}\right) U_{p-1},\left(\begin{array}{l}
1 \\
0
\end{array}\right) U_{p-1} C(g)+\left(\begin{array}{l}
1 \\
1
\end{array}\right) U_{p-2}, \cdots,\left(\begin{array}{c}
m-1 \\
0
\end{array}\right) U_{p-1} C^{m-1}(g) \\
+\left(\begin{array}{c}
m-1 \\
1
\end{array}\right) U_{p-2} C^{m-2}(g)+\cdots+\left(\begin{array}{c}
m-1 \\
p-2
\end{array}\right) U_{1} C^{m-p+1}(g)
\end{array}
$$

where

$$
\begin{aligned}
& U_{p-1} f[C(g)]+U_{p-2} f^{\prime}[C(g)]+\cdots+U_{1} \frac{f^{(p-2)}[C(g)]}{(p-2) !}=0, \\
& U_{p-2} f[C(g)]+U_{p-3} f^{\prime}[C(g)]+\cdots+U_{1} \frac{f^{(p-3)}[C(g)]}{(p-3) !}=0, \\
& \quad \cdot \\
& \quad \\
& U_{1} f[C(g)]=0 .
\end{aligned}
$$

Now set $C(f) X_{p}=X_{p} C(g)+X_{p-1}$. If the rows of $X_{p}$ are $V_{1}=U_{p}$, $V_{2}, \cdots, V_{m}$, then the rows of the left side of (7) are $V_{2}, V_{3}, \cdots, V_{m}$ and $\sum_{i=1}^{m} a_{i} V_{i}$. The rows of the right side of (7) are

$$
\begin{aligned}
V_{1} C(g)+\left(\begin{array}{l}
0 \\
0
\end{array}\right) U_{p-1}= & U_{p} C(g)+\left(\begin{array}{l}
0 \\
0
\end{array}\right) U_{p-1}, \\
& V_{2} C(g)+\left(\begin{array}{l}
1 \\
0
\end{array}\right) U_{p-1} C(g)+\left(\begin{array}{l}
1 \\
1
\end{array}\right) U_{p-2}, \cdots \\
& V_{m} C(g)+\left(\begin{array}{c}
m-1 \\
0
\end{array}\right) U_{p-1} C^{m-1}(g)+\cdots \\
& +\left(\begin{array}{c}
m-1 \\
p-2
\end{array}\right) U_{1} C^{m-p+1}(g) .
\end{aligned}
$$


According to Theorem 1, the rows of $X_{p}$ are

$$
\begin{aligned}
\left(\begin{array}{l}
0 \\
0
\end{array}\right) U_{p},\left(\begin{array}{l}
1 \\
0
\end{array}\right) U_{p} C(g) & +\left(\begin{array}{l}
1 \\
1
\end{array}\right) U_{p-1}, \cdots,\left(\begin{array}{c}
m-1 \\
0
\end{array}\right) U_{\nu} C^{m-1}(g) \\
& +\left(\begin{array}{c}
m-1 \\
1
\end{array}\right) U_{p-1} C^{m-2}(g)+\cdots+\left(\begin{array}{c}
m-1 \\
p-1
\end{array}\right) U_{1} C^{m-p}(g)
\end{aligned}
$$

and

$$
\begin{aligned}
\sum_{i=1}^{m} a_{i} V_{i}=\left(\begin{array}{c}
m \\
0
\end{array}\right) U_{p} C^{m}(g)+\left(\begin{array}{c}
m \\
1
\end{array}\right) U_{\nu-1} C^{n-1}(g) & +\cdots \\
& +\left(\begin{array}{c}
m \\
p-1
\end{array}\right) U_{1} C^{m-p+1}(g)
\end{aligned}
$$

by setting the corresponding rows of both sides of (7) equal. Replacing $V_{i}$ by $V_{i}=\sum_{j=0}^{i-1}\left(\begin{array}{c}i-1 \\ 0\end{array}\right) U_{p-j} C^{i-j-1}(g), \quad i=2,3, \cdots, m$, where $U_{p-j}=0$ if $j>p$, we have

$$
\begin{aligned}
& \sum_{i=1}^{m} a_{i} V_{i}= a_{1} U_{p}+a_{2}\left[U_{p} C(g)+\left(\begin{array}{l}
1 \\
1
\end{array}\right) U_{p-1}\right] \\
&+\cdots \\
&+a_{m}\left[\left(\begin{array}{c}
m-1 \\
0
\end{array}\right) U_{p} C^{m-1}(g)+\left(\begin{array}{c}
m-1 \\
1
\end{array}\right) U_{p-1} C^{m-2}(g)+\cdots\right. \\
&\left.+\left(\begin{array}{c}
m-1 \\
p-1
\end{array}\right) U_{1} C^{m-p}(g)\right] \\
&=\left(\begin{array}{c}
m \\
0
\end{array}\right) U_{p} C^{m}(g)+\left(\begin{array}{c}
m \\
1
\end{array}\right) U_{p-1} C^{m-1}(g) \\
&+\cdots \\
&+\left(\begin{array}{c}
m \\
p-1
\end{array}\right) U_{1} C^{m-p+1}(g) .
\end{aligned}
$$

Transposing and collecting the coefficients of $U_{p}, U_{p-1}, \cdots, U_{1}$ we get

$$
U_{p} f[C(g)]+U_{p-1} f^{\prime}[C(g)]+\cdots+U_{1} \frac{f^{(p-1)}[C(g)]}{(p-1) !}=0 .
$$

Since all the steps of this proof are reversible, the converse holds.

It should be noted that if $g(x)$ divides $f(x)$, then

$$
\begin{gathered}
U_{p} f[C(g)]=0, \\
U_{p-1} f[C(g)]=0, \\
\cdot \\
\cdot \\
U_{1} f[C(g)]=0
\end{gathered}
$$

and $U_{p}$ is arbitrary. 
Equations (6) are satisfied if and only if $U_{k}[C(g)] f[C(g)]+U_{k-1}[C(g)] f^{\prime}[C(g)]+\cdots$

$$
\begin{aligned}
& +U_{1}[C(g)] \frac{f^{(k-1)}[C(g)]}{(k-1) !}=0, \\
& U_{k-1}[C(g)] f[C(g)]+U_{k-2}[C(g)] f^{\prime}[C(g)]+\cdots \\
& +U_{1}[C(g)] \frac{f^{(k-2)}[C(g)]}{(k-2) !}=0, \\
& U_{1}[C(g)] f[C(g)]=0,
\end{aligned}
$$

thus $U_{1}, U_{2}, \cdots, U_{k-1}$ must be such that

$$
\begin{aligned}
& U_{1}(x) f(x)=g(x) k_{1}(x), \\
& U_{2}(x) f(x)+U_{1}(x) f^{\prime}(x)=g(x) k_{2}(x), \\
& \cdot \\
& \cdot \\
& U_{k-1}(x) f(x)+U_{k-2}(x) f^{\prime}(x)+\cdots+U_{1}(x) \frac{f^{(k-2)}(x)}{(k-2) !}=g(x) k_{p-1}(x),
\end{aligned}
$$

where $k_{i}(x)$ for $i=1,2, \cdots, p-1$ are arbitrary polynomials such that the degree of $g(x) k_{i}(x)$ is not greater than $m+n-2$. Therefore the set of $1 \times n$ matrices $U_{1}, U_{2}, \cdots, U_{k-1}$ are obtained through their associated polynomials.

\section{BIBLIOGRAPHY}

1. W. V. Parker, The matrix equation $A X=X B$, Duke Math. J., 17 (1950), 43-51. MR 11, 412.

2. - Matrices and polynomials, Amer. Math. Monthly 61 (1954), 182-183. MR 15, 672.

3. W. V. Parker and J. C. Eaves, Matrices, Ronald Press, New York, 1960. MR 21 \#6373.

4. William E. Roth, On K-commutative matrices, Trans. Amer. Math. Soc. 39 (1936), 483-495.

5. Edmond Dale Dixon, Matric polynomials which are higher commutators, Pacific J. Math. 32 (1970), 55-63. MR 40 \#7283.

Department of Mathematics, Tennessee Technological University, Cookeville, TENNESSEE 38501 